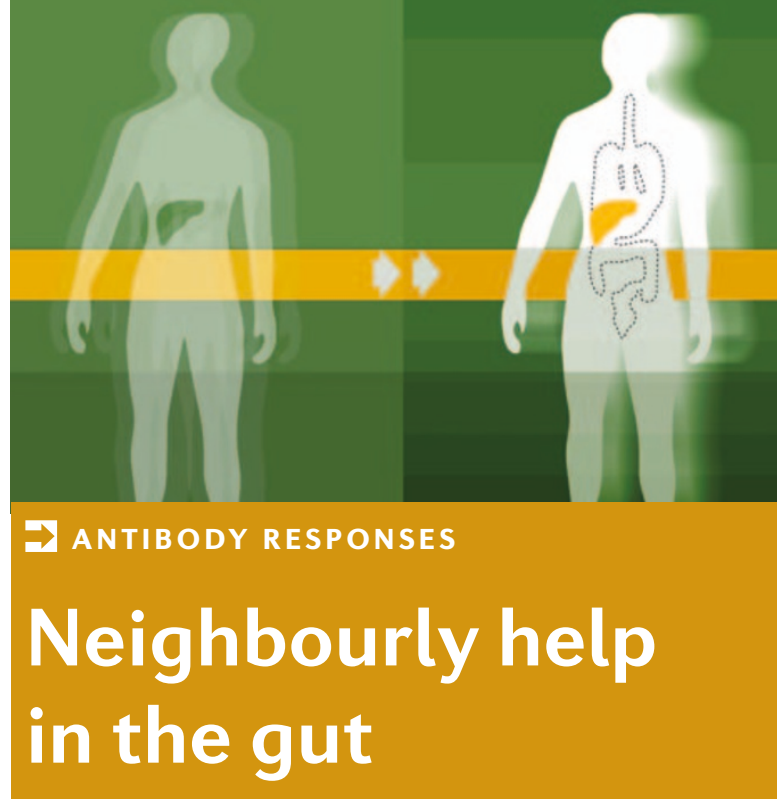

Most of the $\lg A$-secreting cells in the body are located in the intestine, and it is thought that the induction of class switching and targeting of these cells to the gut depends on signals from the gut-associated lymphoid tissue (GALT). But what are the signals that make a B cell home to the gut and specifically produce lgA? Mora et al. now show that the vitamin A metabolite retinoic acid produced by GALT-derived dendritic cells (DCs) confers gut tropism and, together with interleukin-6 (IL-6) or IL-5, induces IgA secretion.

DCs in the GALT, unlike DCs found in other lymphoid tissues, synthesize retinoic acid, which has been shown to induce the expression of a distinct set of trafficking molecules on the surface of T cells that infiltrate the gut. So, the authors examined the effect of GALT-derived DCs on expression of the gut-specific trafficking molecules $\alpha_{4} \beta_{7}$-integrin and CC-chemokine receptor 9 (CCR9) on B cells. Co-culture of $B$ cells with DCs derived from Peyer's patches resulted in $\alpha_{4} \beta_{7}$-integrin ${ }^{\text {hi }} C C R 9^{\text {hi }} B$ cells. By contrast, co-culture of $B$ cells with DCs isolated from peripheral lymph nodes induced $\alpha_{4} \beta_{7}$-integrin ${ }^{\text {low }} C C R 9^{\text {low }} B$ cells. Interestingly, expression of these homing molecules by $B$ cells could be induced, in the absence of DCs, by exogenous retinoic acid. Therefore, only DCs from the GALT, through the secretion of retinoic acid, induce gut-tropic $B$ cells.

The authors then examined the ability of these $B$ cells to produce lgA. Only Peyer's patch-derived DCs induced de novo class switching and secretion of $\lg A$ by $B$ cells. Blocking of the retinoic acid receptor with the receptor antagonist LE540 decreased IgA secretion in these co-cultures. However, retinoic acid alone was not sufficient to induce $\lg A$ secretion, indicating that, although retinoic acid is involved, additional factors are required. Blocking of IL- 6 or IL-5 (cytokines that are known to induce $\lg$ A responses) with specific antibodies, together with LE540 (but not on their own), lead to a reduction in $\lg A$ secretion.

So, these data show that GALT-derived DCs directly induce gut tropism in a retinoic-acid-dependent manner, and they induce class switching to $\lg \mathrm{A}$ in $\mathrm{B}$ cells through a synergistic mechanism that also involves retinoic acid. This study is of clinical relevance as it might explain why vitamin A deficiency exacerbates diarrhoeal disease in malnourished children.

Olive Leavy

ORIGINAL RESEARCH PAPER Mora, J. R. et al. Generation of gut-homing IgA-secreting B cells by intestinal dendritic cells. Science 314, 1157-1160 (2006)

\section{A stimulating collaboration}

The catalogue of molecules that are involved in activating or inhibiting T-cell function is ever increasing, and now another can be added to it. In a study published in The Journal of Immunology, NGFI-A-binding protein 2 (NAB2) is shown to function as a co-activator of T-cell function by promoting the transcription of interleukin-2 (IL2) mediated by the transcription factor early growth response 1 (EGR1).

NAB2 has no DNA-binding domain so it must collaborate gene transcription. In this capacity, it has been shown to be both a coactivator and a co-repressor of EGRmediated transcription. In T cells, expression of the EGR proteins EGR1, EGR2 and EGR3 is markedly upregulated after ligation of the T-cell with transcription factors to affect receptor (TCR). EGR1 is known to stimulate T-cell activation and promote IL-2 production, whereas EGR2 and EGR3 have inhibitory effects on T cells. So, how does NAB2 influence EGR-mediated transcription in $\mathrm{T}$ cells?

Initial analysis of NAB2 expression revealed that the highest levels of NAB2 protein expression coincided with maximum IL2 transcription in T cells stimulated with CD3- and CD28-specific antibodies. This led the authors to suggest that NAB2 might have an activating role in $\mathrm{T}$ cells. Consistent with this hypothesis, overexpression of NAB2 increased the transcription of a reporter gene driven by the IL2 promoter. By contrast, knockdown of NAB2 expression using small interfering RNA led to a
$\Rightarrow$ DENDRITIC CELLS

\section{Terminal differentiation of plasmacytoid dendritic cells}

Plasmacytoid dendritic cells (pDCs) share several features with conventional dendritic cells (cDCs) but they are unique in their ability to produce large amounts of type I interferons in response to viral infection. However, the signals that drive pDC differentiation are not clearly understood. Now, the laboratory of Susan Chan and Philippe Kastner reports in Blood that the zinc-finger transcription factor Ikaros is required for the generation of peripheral pDCs from a committed pDC population in the bone marrow.
Ikaros is essential for the development of multiple haematopoietic lineages. Previous studies have shown that expression of a dominant-negative form of lkaros resulted in the lack of both $C D 8 \alpha^{+}$and $C D 8 \alpha^{-}$subsets of cDCs. By contrast, an lkaros null mutation resulted in selective loss of the $\mathrm{CD} 8 \alpha^{-}$subset of $\mathrm{cDC}$ s. In this study, Chan and colleagues studied mice expressing a hypomorphic mutant of Ikaros (Ik $\left.\mathrm{k}^{\mathrm{L} / L}\right)$ which resulted in lowlevel expression of lkaros protein in haematopoietic cells. Using the

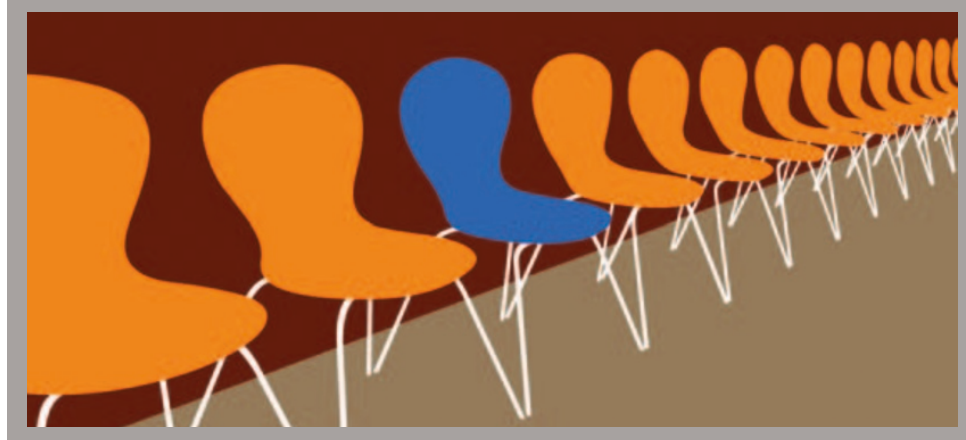




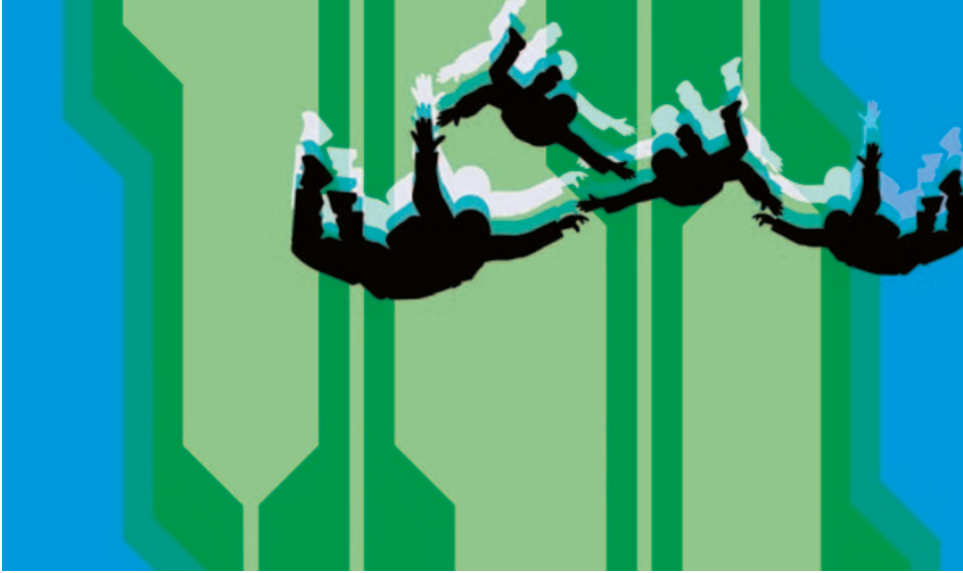

reduction in both proliferation and IL-2 production by Jurkat T cells activated by ligation of the TCR and the co-stimulatory molecule CD28. Finally, Jurkat T cells expressing a dominant-negative form of $\mathrm{NAB} 2$ that lacks the NCD2 domain responsible for interacting with EGR proteins inhibited the induction of IL2-promoter-driven transcription by NAB2.

The authors next asked whether the activating effect of NAB2 was mediated through EGR1, which is known to bind the IL2 promoter and promote IL2 transcription. By carrying out chromatin immuno-

precipitation assays, they showed that after T-cell activation both NAB2 and EGR1 are recruited to the IL 2 promoter. Importantly, the recruitment of both EGR1 and NAB1 was abrogated by mutation of the EGR1-binding site in the IL2 promoter, which confirms that NAB2 binds to the IL2 promoter by interacting with EGR1.

So, this study identifies NAB2 as a new TCR-induced transcriptional co-activator of IL-2 production.

ORIGINAL RESEARCH PAPER Collins, S. et al. TCR-induced NAB2 enhances T cell function by coactivating IL-2 transcription. J. Immunol. 177, 8301-8305 (2006)
Lucy Bird

pDC-specific antibody $120 \mathrm{G} 8$, they showed that there is selective loss of the peripheral $\mathrm{pDC}$ population in these mice. To demonstrate functional deficiency, the authors carried out in vitro and in vivo assays using stimuli (including $\mathrm{CpG}$ oligonucleotides, which bind to Toll-like receptor 9 (TLR9) and R848, a ligand for TLR7) known to drive $\mathrm{pDC}$ s to produce interferon- $\alpha$ (IFN $\alpha$ ) and interleukin-12 (IL-12). pDCs from Ik $\mathrm{K}^{\mathrm{L} / \mathrm{L}}$ mice produced IL-12 but not IFN $\alpha$ in response to stimulation. By contrast, cDCs produced both IFN $\alpha$ and IL-12, showing that their responses are not significantly affected in $1 \mathrm{k}^{\mathrm{L} / \mathrm{L}}$ mice. This functional defect in the pDCs rendered the $\mathrm{Ik}^{\mathrm{L} / \mathrm{L}}$ mice susceptible to murine cytomegalovirus infection, in which pDCs are thought to be the main producer of the protective type I interferons.

pDC development occurs in the bone marrow and when the authors used the antibody $120 \mathrm{G} 8$ to look in the bone marrow, they identified a population of pDCs in $1 k^{1 / L}$ mice. These cells produced IFN $\alpha$ in response to stimulation with $\mathrm{CpG}$ oligonucleotides and influenza virus, but they were not terminally differentiated, even in the presence of FLT3 (FMS-related tyrosine kinase 3) ligand, which directs the development of pDCs from haematopoietic stem cells in the bone marrow.

To determine whether the defect is intrinsic to the $\mathrm{pDC}$ s or affects another haematopoietic population, the authors generated bone-marrow chimaeras. The $\mathrm{Ik}^{\mathrm{L} / \mathrm{L}} \mathrm{pDC}$ s did not terminally differentiate even when placed in a wild-type, bone-marrow and stromal-cell environment, confirming that the reduced expression of $\mathrm{lkaros}$ in $\mathrm{I} \mathrm{k}^{\mathrm{L} / \mathrm{L}}$ mice directly influences pDC development. Ikaros is considered to be a transcriptional repressor, and results from gene expression profiling experiments showed that 370 genes were strongly overexpressed in $\mathrm{pDC}$ s from $\mathrm{lk}^{\mathrm{L} / \mathrm{L}}$ mice, indicating that lkaros acts in wild-type $\mathrm{pDC}$ s by silencing a wide range of genes.

This study indicates that lkaros is crucial for terminal differentiation of pDCs. Although other haematopoietic lineages are affected by the $\mathrm{k}^{\mathrm{L} / \mathrm{L}}$ mutation, $\mathrm{Ik}^{\mathrm{L} / \mathrm{L}}$ mice might provide a useful model for studying in vivo immune responses to pathogens in the absence of pDCs.

ORIGINAL RESEARCH PAPER Allman, D. et al. Ikaros is required for plasmacytoid dendritic cell differentiation. Blood 108, 4025-4034 (2006)
Elaine Bell

\section{RESEARCH HIGHLIGHTS}

\section{IN BRIEF}

\section{DIYMPHOCYTE MIGRATION}

\section{Orchestration of lymphocyte chemotaxis by}

mitochondrial dynamics.

Campello, S. et al. J. Exp. Med. 4 December 2006 (doi:10.1084/ jem.20061877)

Migration of lymphocytes to sites of inflammation is an integral part of the immune reaction. For directed migration, the cell must undergo cellular polarization to form a leading edge and a trailing uropod. But what is the role of mitochondria in this process? Campello et al. examined the redistribution of mitochondria during cell migration and found that the mitochondria specifically concentrate at the uropod. They are transported along the microtubules through a process that requires $\mathrm{G}$-protein signalling and involves an alteration of their shape. By interfering with the proteins that regulate mitochondrial shape through fission and fusion, the authors showed that mitochondrial fission was necessary for their relocation to the uropod and for promoting lymphocyte migration, whereas mitochondrial fusion inhibited both processes. This study shows that accumulation of mitochondria at the uropod is required to regulate the motor of migrating lymphocytes.

\section{$\Rightarrow$ NATURAL KILLER CELLS}

Cross-talk with myeloid accessory cells regulates human natural killer cell interferon- $\gamma$ responses to malaria.

\section{Newman, K. et al. PLoS Pathogens 2, e118 (2006)}

The early production of interferon- $\gamma$ (IFN $\gamma$ ) by natural killer (NK) cells after exposure to Plasmodium falciparum-infected red blood cells (RBCs) is an important element of the cell-mediated effector response to malaria infection. However, it is thought that optimal NK-cell responses require signals from accessory cells. Here Riley and colleagues show that both contact-dependent and cytokine-derived signals from monocytes and myeloid dendritic cells are essential for early IFN $\gamma$ production by NK cells. Interestingly, the capacity of NK cells to produce IFN $\gamma$ correlated with the expression of co-stimulatory molecules on resting accessory cells. These accessory cells are also activated after exposure to infected RBCs, and NK cells are required to maintain their maturation status. So, reciprocal interactions between NK cells and accessory cells can determine the magnitude of the immune response to P. falciparum.

\section{$\Rightarrow$ T-CELL MIGRATION}

\section{Physiological and aberrant regulation of memory} T cell trafficking by the co-stimulatory molecule CD28.

Mirenda, V. et al. Blood 21 November 2006 (doi:10.1182/blood-200610-050724)

It is well known that CD28 is a co-stimulatory molecule for antigen-induced T-cell activation. But does CD28 signalling have any other physiological functions? Here the authors showed that activation of CD28 on T cells induced integrin clustering and increased integrin-mediated migration in vitro. Engagement of CD28 in vivo led to the migration of memory T cells to non-lymphoid sites, which was independent of T-cell-receptorderived signals and expression of specific homing molecules. This is in contrast to cytotoxic T-lymphocyte antigen 4 (CTLA4)mediated signals, which prevent tissue infiltration by memory T cells. This new physiological function of CD28 has important implications for targeting CD28 in human disease therapy. 